\title{
Power Transmission Determined by the Mutual Impedance and the Transducer Power Gain in the Near Field Region
}

\author{
Che-Young Kim
}

\begin{abstract}
This paper describes the role of mutual impedance and the transducer power gain which comes from key parameters to determine the amount of wireless power especially in a near-field environment. These two key parameters are applied to the two configurations; one is a dipole-dipole, and the other is a dipole-metal plate-loop configuration. Discussions are given on the achievable maximum power transfer between the sender and the receiver affected by the matching and the pass blockage.
\end{abstract}

Key words: Wireless Power Transfer, Mutual Impedance, Transducer Power Gain, Matching.

\section{Introduction}

Depending on the relative wavelength distance between a sender and a receiver, wave regions are classified as near field and far field regions [1]. When a pair of antenna operates in the far field region, mutual impedance becomes so negligible that each transmitting and receiving antenna could be treated independently and so little attention might be paid on their mutual impedance. However, for antennas operated in the near field, mutual impedance could no longer be ignored since the antenna pair becomes very dependent on each other. This fact is equally applied to recent wireless power transmissions since most of this system operates in a near field region. RFID (Radio Frequency Identification) is one of example operating in near field zone with the principle of magnetic induction [2]. Many papers are published about these topics [3], [4], including some domestic papers about wireless power transmissions [5], [6].

The main concern of this paper is to show parameters simple and accurate enough to determine the amount of received power from the sender under the near field circumstances. This task is accomplished by introducing the mutual impedance and the transducer power gain. Once the mutual impedance is known either by analytical formula or by simulated value, the transducer power gain can be determined based on the "black box" approach. The resulting transducer gain enables us to calculate the power arrived at the receiving antenna, a key quantity in wireless power transmission.

As an illustration, configurations of \{dipole-dipole\} and \{dipole-metal plate-loop\} are considered. The configuration of \{dipole-dipole\} is included here because its formula of mutual impedance is already known. Therefore one can estimate the errors involved in numerical calculation.

A second configuration having no analytic solution is also studied because we can still calculate the receiving power using the transducer power gain formula even in this situation. In addition, the achievable power transfer is discussed with respect to $50 \mathrm{ohm}$ reference resistance and the conjugate matched load. We focus on the moderate level of analytic approach not on the elaborate experimental study to provide the stepping stones between academic interest and industrial practice.

\section{II . Formulation of the Problem}

\section{2-1 Mutual Impedance of Dipole-Dipole Structure}

Mutual impedance dictates the determination of wireless power, particularly, on systems operating in a near field region. Fig. 1 shows a pair of half wavelength dipoles of radius $a$, length $\ell$ and distance $d$. While one antenna is driven by center feeding, the opposite side antenna receives the incoming power flux from the nearby excited rod. The distance between two antennas varies over $0.1 \lambda_{0}$ to $1 \lambda_{0}$ to maintain the near field, and the free space wavelength $\lambda_{0}$ corresponds to an operating frequency of $1.5 \mathrm{GHz}$. The analytic mutual impedance formula based on EMF (Electromotive Force) method has been presented by Carter for the dipoles in Fig. 1, applicable to zero radius wire [7], [8].

Manuscript received July 21, 2011 ; revised August 25, 2011. (ID No. 20110721-1J)

Department of Electronics, College of IT Engineering, Kyung-pook National University, Daegu, Korea.

Corresponding Author : Che-Young Kim (e-mail : cykim@ee.knu.ac.kr) 


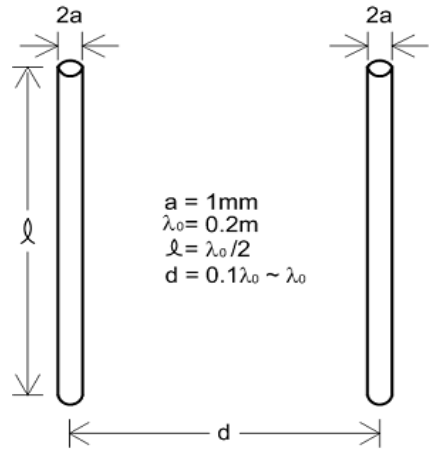

Fig. 1. The two identical dipoles in the dipole-dipole configuration.

$$
\begin{aligned}
& R_{21}=\frac{\eta}{4 \pi}\left[2 C\left(u_{0}\right)-C\left(u_{1}\right)-C\left(u_{2}\right)\right] \\
& X_{21}=-\frac{\eta}{4 \pi}\left[2 S\left(u_{0}\right)-S\left(u_{1}\right)-S\left(u_{2}\right)\right]
\end{aligned}
$$

where

$$
\begin{aligned}
& u_{0}=k_{0} d, \quad u_{1}=k_{0}\left(\sqrt{d^{2}+\ell^{2}}+\ell\right), \\
& u_{2}=k_{0}\left(\sqrt{d^{2}+\ell^{2}}-\ell\right) \\
& C(x)=0.577215665+\ln (x)+\int_{0}^{x} \frac{\cos t-1}{t} d t \\
& S(x)=\int_{0}^{x} \frac{\sin t}{t} d t
\end{aligned}
$$

where $\eta$ denotes characteristic impedance of free space and $k_{0}=2 \pi / \lambda_{0}$. The symbols $R_{21}$ and $X_{21}$ in equations (1) and (2) represent the real and imaginary part of mutual impedance, respectively. It is noted that equations (1) and (2) are restricted to antennas having integer values of half wavelengths with zero radius wire. Thus, some error is unavoidable for the antenna having a finite-sized radius.

The imaginary part of mutual impedance is shown in Fig. 2. In this figure, $\operatorname{Im}\left(Z_{21}\right)$ for the finite-sized radius is a numerical quantity obtained by transforming the scattering parameter to the impedance parameter [9]. $X_{21}$ comes from equation (2). Some disagreement between them is to be expected due to the involvement of zero and finite-sized radii. The numerical difference between them will naturally be decreased as the radius of the antenna gets smaller. However, what matters is not reducing the error itself, but characterizing the related parameters.

\section{2-2 Transducer Power Gain Formula}

The antenna structure can be modeled in terms of a

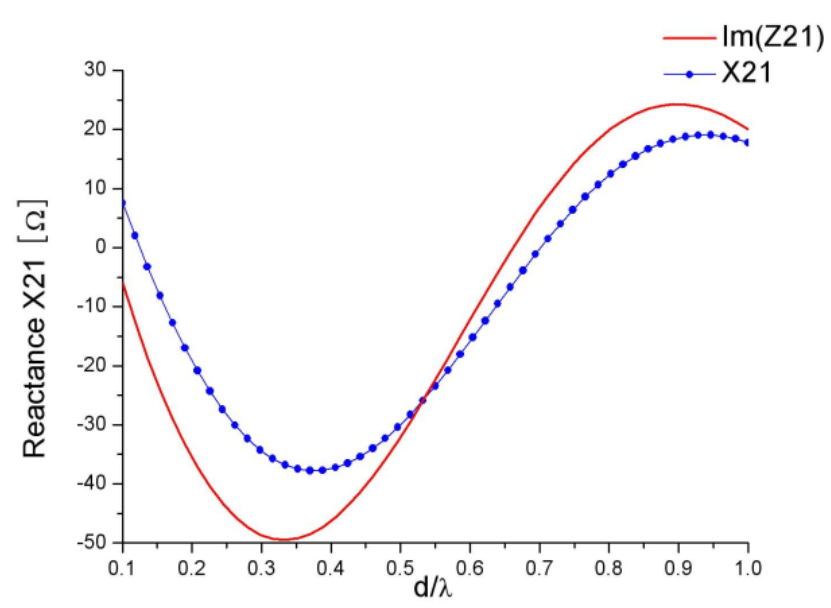

Fig. 2. Reactive part of mutual impedance.

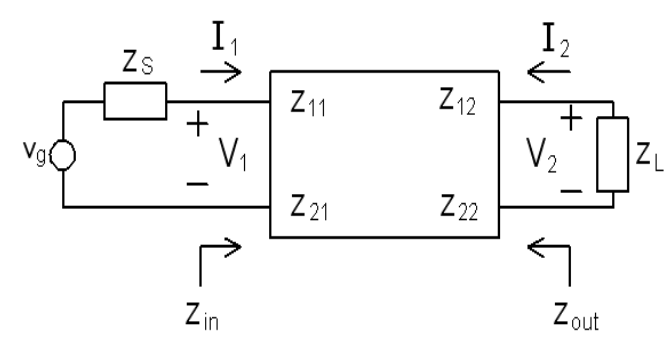

Fig. 3. Antenna structure represented by two-port network.

two port impedance network often regarded as a "black box" approach. Fig. 3 shows a two-port network representing the antenna system seen, for instance, in Fig. 1. Here, $Z_{\mathrm{S}}$ and $Z_{\mathrm{L}}$ are source and load impedance terminating at each port, respectively, and the mutual impedance computed by equations (1) and (2) also appears.

For the two-port network shown in Fig. 3, after solving for $I_{2}$, one may obtain the transducer power gain [10]

$$
G_{T}=\frac{P_{L}}{P_{\text {ave }}}=4 \frac{\left|Z_{21}\right|^{2} \operatorname{Re}\left(Z_{S}\right) \operatorname{Re}\left(Z_{L}\right)}{|D|^{2}}
$$

where

$$
D=\left(Z_{11}+Z_{S}\right)\left(Z_{22}+Z_{L}\right)-Z_{12} Z_{21}
$$

$P_{L}$ represents the delivered power to the load and $P_{\text {ave }}$ is the available source power from the generator. It is worth noting that $G_{T}$ is proportional to the mutual impedance squared, and equation (3) corresponds to the transferred power from the source to the load referred in Kurs [11].

At the input and output ports, the condition of conjugate match is found to be 


$$
\begin{aligned}
& Z_{S}=R_{S}+j X_{S}=Z_{\text {in }}{ }^{*} \\
& Z_{L}=R_{L}+j X_{L}=Z_{\text {out }}{ }^{*}
\end{aligned}
$$

After solving coupled equations (4) and (5) by using the solve block in Mathcad [12], for instance, finalizing the matching network design to produce the maximum power transfer is straightforward.

\section{Computed Results and Discussions}

Fig. 4 shows a structure consisting of dipole, metal plate, and loop antenna. This is an identical dipole as used in Fig. 1. The diameter of the loop wire is $2 \mathrm{~mm}$ equal to that of the dipole antenna. The radius of the loop is set to $\lambda_{0} / 2 \pi$ to resonate the antenna, where $\lambda_{0}=0.2[\mathrm{~m}]$. The metal plate is a square plate with $\lambda_{0}$ on one side with thickness of zero. The middle points of the antennas are aligned with the center of the metal plate.

Because the structures are submerged in a near field region, the input impedance undertakes the influence of mutual impedance presented in Fig. 3. As a result, the input impedance for the load of $50 \mathrm{ohm}$ and for the conjugate load is different from each other, since $Z_{L}$ is reflected to the input impedance via mutual impedance. This, in turn, has an effect on the transducer power gain. Once calculating the $S$-parameters in terms of the current method's code and then transformed into the $Z$-parameters, we find the transducer power gain by using equation (3).

In Fig. 5, transducer power gain $G_{T}$ is plotted in decibel units. Solid line $\left(G_{T}\right)_{\mathrm{z} 0}$ is for a $50 \mathrm{ohm}$ load and dotted line $\left(G_{T}\right)_{\text {opt }}$ is for the conjugate matched load. As can be seen in Fig 5, dotted line $\left(G_{T}\right)_{\text {opt }}$ bounds the upper limit since it is the value under the conjugate matched

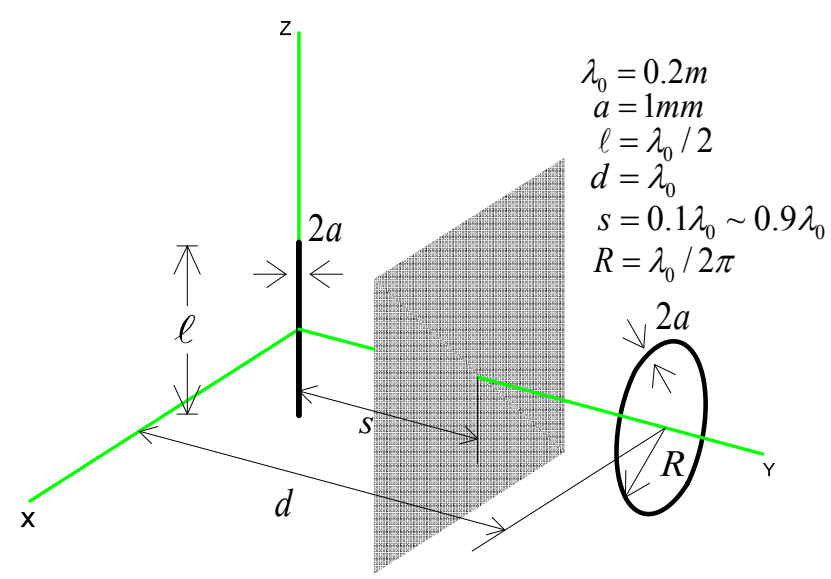

Fig. 4. Dipole-metal plate-loop configuration.

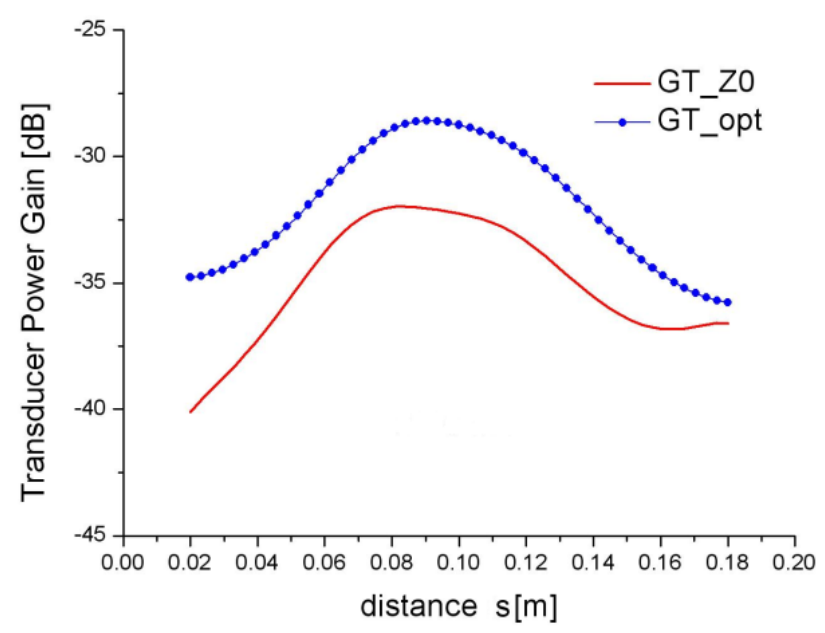

Fig. 5. Transducer power gain versus metal plate placement.

condition. Thus no matching circuit would yield a higher value than $\left(G_{T}\right)_{\text {opt }}$ numerals as indicated.

The metal plate in Fig. 4 is allowed to move between the dipole and the loop antenna. Allowed distance $S$ varies between $0.1 \lambda_{0}$ and $0.9 \lambda_{0}$. Because the metal plate between antennas blocks out the electromagnetic waves, the power reaching the receiving antenna is lower than that of the free space case. This blocking effect clearly appears in Fig. 5. Based on computed $G_{T}$ values, the conjugate matching for a maximum power transfer is quite effective to enhance the level of power reception.

The $G_{T}$ value obtained for conjugate matching is higher than those out of matching with an order of $2 \mathrm{~dB}$ to $5 \mathrm{~dB}$. The corresponding $G_{T}$ value for a dipole-metal plate-dipole \} configuration shows about $5 \mathrm{~dB}$ lower at a deep shadow than that of Fig. 5, though not shown in here. This difference adheres to the predominantly magnetic coupling in a near field region so that the loop becomes more effective than the dipole. As the metal plate locates closer to the dipole or to the loop side, the $G_{T}$ value decreases and reaches toward the top point near the middle position. This phenomenon occurs due to the pass blockage of radio waves in the presence of the metal plate. Whenever the metal plate gets closer to either the dipole or loop a maximum shielding is realized. A minimum shielding occurs, however, if it is located at a middle position. As a result, a sort of shielding effectiveness is also demonstrated in Fig. 5.

\section{Conclusions}

The two essential parameters of mutual impedance and transducer power gain have been introduced and discussed. These parameters play a key role determining 
wireless power transmissions. To provide a state-of-arts description on the role of these parameters, two configurations \{dipole-dipole\} and \{dipole-metal plate-loop\} have been defined and the associated transducer power gain is computed. In a moving plate case, about $2 \mathrm{~dB}$ to $5 \mathrm{~dB}$ more power would be transferred to the load if conjugate matching could be maintained during the plate movement.

This study might be helpful and informative in the design and analysis of wireless power transmission systems operating in near field regions. Furthermore, this article will also be significant for its insight into the influence of mutual impedance on matching circuits and for estimations of system performance.

This research was supported by Kyung-pook National University Research Fund, 2010.

\section{References}

[1] W. L. Stutzman, G. A. Thiele, Antenna Theory and Design, John Wiley \& Sons, Inc., pp. 23-24, 1981.

[2] K. Finkenzeller, RFID Handbook, Wiley, p. 42, 2003.

[3] W. C. Brown, "The history of power transmission by

\section{Che-Young Kim}

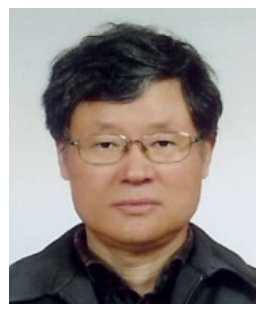

completed his Ph.D. degree in electrical engineering at Korea Advanced Institute of Science and Technology, Seoul in 1990, and received his Bachelor degree in Electronics Engineering at Kyung-pook National University, Daegu in 1976. During 1979 1992, he was an instructor, assistant professor, and associate professor at Kyung-pook National University. Since 1993, he has been a professor at the same university. He was a visiting scholar at Syracuse University from 1985 to 1986 and became a research scientist at Massachusetts Institute of Technology in USA from 1991 to 1993 . He is now serving as one of editors in the Progress in Electromagnetics Research (PIER) and Journal of Electromagnetic Waves and Applications (JEMWA) since 2006. He was awarded the Paper of Year entitled "A propagation model of microcell and picocell in urban environments," listed on Journal of SK Telecommunications Review in June 1996. His technical interests include the basic electromagnetic theory and measurements, wave propagation, metamaterials, and wireless energy transmission. His current research involves the RF filters using the metamaterial technology. radio waves," IEEE Trans. Microwave Theory Tech., vol. 32, no. 9, pp. 1230-1242, Sep. 1984.

[4] Wikipedia, "Wireless energy transfer," http://en.wik ipedia.org/wiki/wireless

[5] K. J. Choi, H. Y. Hwang, "Low-power wireless transmission at $2.45 \mathrm{GHz}$ band," Journal of KIEES, vol. 20, no. 8, pp. 777-783, Aug. 2009.

[6] J. W. Kim, H. H. Ji, Y. G. Choi, Y. H. Yun, K. H. Kim, and Y. J. Park, "Study on arrangement of selfresonant coils in wireless power transfer system based on magnetic resonance," Journal of KIEES, vol. 21, no. 6, pp. 564-572, Jun. 2010.

[7] P. S. Carter, "Circuit relations in radiating systems and applications to antenna problems," Proc. IRE, vol. 20, no. 6, pp. 1004-1041, Jun. 1932.

[8] C. A. Balanis, Antenna Theory, 2nd edition, John Wiley \& Sons, Inc., p. 417, 1997.

[9] D. M. Pozar, Microwave Engineering, 2nd edition, John Wiley \& Sons, Inc., p. 211, 1998.

[10] R. Ludwig, P. Bretchko, RF Circuit Design: Theory and Applications, Prentice Hall, pp. 466-468, 2000.

[11] A. Kurs, A. Karalis, R. Moffatt, J. Joannopoulos, P. Fisher, and M. Soljacic, "Wireless power transfer via strongly coupled magnetic resonances," Science, vol. 317, pp. 83-86, 2007.

[12] http://www.mathsoft.com 